The LaNCet,] MR. H. LEE ON THE TREATMENT OF ANEURISM BY ACUPRESSURE. [Nov. 10, 1866. 519

rises, tilting up the lever as already described. Although the carotid pulse-curve exhibits great variety in different individuals, its form always indicates that at each stroke of the heart the lever rises abruptly, remains elevated for a short period, and then falls as suddenly as it rose. That the period of elevation coincides with the actual influx of blood from the ventricle is proved by experiments made by Chauveau, who found that when his beautiful instrument for measuring the relocity of the blood-stream was introduced into the carotid of a horse, to which a sphygmograph was at the same time applied, the acceleration of the current corresponded in degree and duration with the distension of the artery.

8. Of the less constant characters of the carotid tracing, some are directly dependent on the mode in which the ventricle contracts, others on peculiarities in the closure of the valves. Under certain conditions, the nature of which cannot be here discussed, the mass of blood in the ventricle is thrown into rapid vibration during the act of contraction. Whenever this is the case the vibration is communicated to the arteries, and shows itself in the carotid tracing; the ascending limb of the pulse-curve being interrupted by a series of notches, each of which corresponds to a vibration. When these vibrations are very well marked, they communicate the sensation of thrill to the finger. The other variations to which reference has been made have to do with the descent of the lever at the end of the systolic period. In some cases this descent is well marked, and is denoted by a deep noteh, as in Fig. 3 ; in others it is marked by axmere change of direction. These differences may be clearly shown to be dependent on the degree to which aortic regurgitation takes place, or, in other words, on the rapidity with which the sigmoid valves close. When the mean arterial pressure is high, the valves close instantaneously; in the contrary condition, when the arteries are flaccid, the valves remain open for an appreciable period after the ventricular contraction has ceased.

9. The preceding observations tend to show that in the great vessels pulsation is produced by a simultaneous increase of tension and acceleration of movement. In the more distant arteries these two effects are no longer coincident. Both aro transmitted; but inasmuch as their propagation is influenced in a directly opposite manner by those physical conditions which exist in the arteries, they tend to separate themselves more and more from each other the further the exploration is made from the heart. Just as a ray of light becomes two by being transmitted through a medium which refracts them at different angles, so the pulse, in its propagation from the heart to the periphery, splits into its two elements of expansion and acceleration.

10. The limits of this paper do not permit me to show the light which these considerations throw on the explanation of the peculiarity and characters of the radial pulse. In antici pation of my next paper, I place before the reader the following summary of the conclusions $I$ hare arrived at with respect to it.

a. The propagation of the systolic acceleration, if it occur at all, gives rise to a rapid expansion of the arterr, which is synchronical with the ventricular systole, and is followed immediately by collapse.

$b$. The propagation of the systolic pressure-wave gives rise to a gradual expansion of the artery, and, in accordance with Weber's law, follows the systole after a variable interval.

$c$. When the resistance due to the mean arterial tension is considerable, the acceleration extends only to the great vessels, so that its effects at the wrist are imperceptible. In such a case the form of the pulse is wave-like. In the opposite condition as regards arterial tension, the effects due to the systolic acceleration are well marked; the elevation of the lever is abrupt, but of short duration.

In my next paper I will endeavour to show how the various forms of pulse-curve which are met with, as well in health as in disease, arise out of the combination of these two effects in various proportions.

Imperial Hoyour to the Profession. - It has been remarked that nearly every profession but that of medicine was represented in the French Senate. This anomaly has struck the Emperor, it would appear, as the Evenement announces that his Majesty's physician, Dr. Conneau, is to be promoted forthwith to a seat in the Luxembourg.

THE decease is announced of Lady Holland, wife of Sir Henry Holland, and daughter of Sydney Smith, of whose brilliant wit and literary power she possessed an hereditary share.

\section{ON THE TREATMENT OF ANEURISM BY} ACUPRESSURE.

\author{
Bi HENRT LEE, EsQ, F.R.C.S.,
} SERGEON TO ST. GIORGE'S HOSPITAL.

Ir is an opinion very generally entertained that the primary object in the treatment of ancurism should be to retard the circulation of the blood throngh the tumour, so as thereby to increase the quantity of coagulum in the sac.* Two propositions are here taken for granted: (1) that the coagulation depends upon the slowness of the motion of the blood; (2) that the eure is in some way associated with the amount of the coagulum. Lpon each of these subjects I would offer a few very brief observations.

1. Is it true that slowness of motion is the essential condition which favours or induces coagulation of the blood in the living vessels? Hunter tied the carotid artery of an animal in two places; the intervening portion of the ressel must have been full of blood, yet when the animal was killed some days afterwards, a coagalum was found idjacent to one of the ligatures only. The preparation is preserved in the museum of the College of Surgeons. I have placed a ligature upon the jugular vein of an ass, and had the animal killed at the expiration of forty-eight hours. On examining the parts, I found only a very small, loose, floating coagulum in the vein above the ligature : there was no appearance of any clot having adhered to the lining membrane of the vein, nor was there any deposit of fibrin upon it. On the other hand, when blood is withdrawn from the body and beaten with a twig, the rapidity with which the twig is moved does not prevent the separation of the fibrin; or, again, if some mercury be shaken with some recently drawn blood, however briskly, the fibrin will adhere to the moving particles of mercury. These instances will, I think, be sufficient to show that retardation in itself is not sufficient to account for the coagulation of the blood, or for the separation of its fibrin in the living body.

2 . Is the cure of an aneurism favoured by a large amount of coagulum?

Hunter thus correctly describes the appearances of a coagulum in an aneurismal sac:- "The firmness and colour of the laminæ in different parts of the tumour are such that it is easy to distinguish an old coagulum from a new one: the external laminæe are of a dusky-brown colour, and these laminæ grow gradually redder as we advance inward toward the current of the blood." t He also says that an aneurism arrived at this state generally yields to the force of the circulation. The fact of the inner laminæ of the coagulum being, as he describes them, of a reddish colour, shows that they approach in their composition to ordinary blood-clots, which are very little fitted to perform the duties of permanent repair. Any amount of this material, which can neither become organised nor absorbed, may serve to prevent farther distension of the sac, but cannot assist in the vital union of diseased or injured parts.

In order to understand the real intention of the deposit of fibrin in injured or discased arteries, we must look to what occurs when the injury is first inllicted, or the disease first commences. At that time we never have a large amount of fibrin deposited. What we see in examining the bodies of those who die of aneurism is the product of long-continued growth. The successive laminæ mark the different periods at which fresh portions of fibrin have been cleposited; but as they have been so deposited the layers first formed have become distended before the impulse of the blood. This impulse, the last formed layers, according to the lescription above given, are still less likely to resist than the first; and so the disease has a natural tendency to increase.

Now, it is a remarkable fact that in animals, wounds of arteries do not produce aneurisms. We learn from this that there is a natural power by which a wounded artery may be repaired; and we nay learn also what the process is by which that restoration is effected.

It has already heen stated that the blood does not coagulate or part with its fibrin readily in the living vessels, but that it does so readily when in presence of any foreign body. A wounded or diseased artery presents such a foreign surface to the blood ; and however rapidly the blood flows over that surface, it will leave some fibrin adbering to it. The fibrin so deposited in the case of a wounderl arterv acts as a temporary $\frac{\text { posited in the case of a wonnderl artery acts }}{\text { * Sie Holmes's Surgery, rol. ni., p. .62. }}$ 
bond of union between the dirided edges. It mechanically glues them together, and, if not disturbed, serres as the bed in which the process of repair is effected. Increased cell growth takes place in the divided edges of the vessel, and this gradually invades the layer of fibrin which serves as the temporary bond of union, until the liring cells from opposite sides unite. This is union by first intention. The laver of fibrin in this process becomes absorbed, probably furmishing a pabulum to the cells which grow into it. In a few rare cases the tibrin itself would appear to become organised; but this is an exception to the general rule.

The union of a divided artery and of a divided vein is thus conducted on the same principles, and it is evident that a small quantity of fibrin only is required for the due performance of the process. As soon as sufficient is deposited to unite the divided edges (and this is sure to take place while the blood retains its natural properties), any additional deposit only embarrasses the operation.

In healthy states of the body, wounds of arteries and veins are alike healed in this manner. But it may happen in both that the process is interfered with. In the case of an artery, the temporary bond of union may as soon as it is formed yield before the impulse of the blood. The intention is then not carried out. A fresh attempt is made; a fresh layer of fibrin is deposited, and this in its turn may yield in a similar way; and thus an aneurism is formed, the union by first intention being frustrated by mechanical means. After an aneurism has attained any size, the quantity of fibrin not only prevents the divided coats of an artery from coming together, but actually tends to pull them apart. The opening into the artery is generally much smaller than the diameter of the tumour, and as the outer layers of fibrin are forced outward, they tend to widen the aperture into the vessel. Now, the blood flows almost as quickly through the veins as through the arteries, and yet we do not find aneurisms in veins. In order that the divided coats of an artery may unite in the same way as those of a vein, it is only necessary that they should be placed under the same circumstances. The essential condition is that the layer of fibrin which unites the divided edges should be left at rest until it has acquired sufficient consistency to resist any mechanical causes of disturbance to which the part may be subjected. In this mode of union it is only necessary that the fibrin deposited should be of proper quality, and should not be disturbed. Any large deposit of fibrin, whether in arteries or veins, is an indication of imperfect action. It shows that the original intention having to a greater or less extent failed, successive subsequent attempts have been made to accomplish the same object. When the healthy natural process of union by first intention takes place, the calibre of the vessel is not obstructed ; but in some cases, where the quality of the blood is altered, or where it has become mixed with some other matter, a coagulum of a greater or less extent will form within an artery, and occasionally this will entirely obliterate its canal. Sir Wm. Fergusson showed me some years ago an instance in which he had pressed the coagulum out of an aneurismal sac, and in which the distal arteries in consequence ceased to pulsate. If in such a case the blood were to coagulate around the fibrin so as to obstruct the canal of the vesse for any length of time, a permanent cure of an aneurism situated upon the obstructed vessel might be expected; but, as I have said, with healthy blood it is very rarely that such coagula form within the cavities of arteries which are not diseased.

The statements that I have now made carry conviction to my own mind that neither slowness of motion of the blood, nor any large quantity of coagulum, is necessary for the due performance of the process of union. It takes place commonly and readily in veins even after a wound has been opened several times, without any obstruction to the circulation; and all that is requisite in order that it may in like manner take place in arteries is that the circumstances may be similar. Thus it is not retardation of the circulation, nor the quantity of fibrin deposited, that is essential for the cure of an aneurism; but some mode of preventing the impulse of the blood upon the newly-formed adhesions: in other words, apposition and rest. These objects may be attained in various ways. Whether intentionally or not, all the operations for aneurism which have been introduced, since the severer operation of amputation, as recommended by Pott, and that of opening the sac as advised by the older surgeons have been abandoned, tend to favour these conditions. What has been termed the Hunterian operation does so in a marked degree: the coagulum is left undisturbed, and the impulse is taken off the injured vessel. Instrumental compression, which has lately been used with a considerable amount of success, has evidently the same effect.
This practice, as old as the time of Hunter, failed at first from the fact that those who attempted it tried to stop the current of the circulation, and thus produced a degree of pain which it was impossible for the patient to bear. With digital pressure the effect is essentially the same, but the degree of compression must necessarily be constantly varying.

The treatment by flexion is a discovery due to Mr. Ernest Hart. The sac, and consequently its contents, are prevented from being distended by being pressed upon in all directions by surrounding structures. The impulse of the blood, by the compression of the tumour against the upper part of the artery, is at the same time diminished.

Thus in all these methods of treatment the same essential conditions are provided for-.viz., rest, and apposition more or less direct of the diseased or divided coats of the artery. It is true that cures have been effected in other ways. Thus the coagulum has been rendered so firm by galvanic action, or by the injection of the perchloride of iron, that it has not yielded. before the pulse of the heart; and this is to do by artificial means that which is naturally done in animals. And instances where this mode of treatment has been successful does not in the least militate against the necessity of the two conditions upon which I have insisted.

Now, in the various plans of treatment which I have mentioned, we may observe a gradual progress towards the accomplishment of the same end by more simpie means. The old operation involving the opening of the sac was succeeded by modifications of the Hunterian operation. This, in many cases, was superseded by various modes of compression; and this again by the less painful and less tedious plan of acute flexion.

I have now the pleasure of submitting to the consideration of the profession what, I believe, may prove a still more simple. and more certain plan of treatment, at least in some casesviz., the treatment by acupressure. I cannot but remember that six years ago $I$ had the pleasure of reading before the Medical Society of London a paper, which was subsequently published as a separate essay. In this I advocated acupressure in certain operations upon the veins-a mode of proceeding which I had at that time practised for a period of seven years. In performing the operation for varicocele it had occurred to me occasionally to wound one of the branches of the spermatic artery, which was not controlled by the needles already introduced. Arterial hæmorrhage would then take place; but this was always commanded by introducing another needle, so as to compress the bleeding vessel. During this period a case occurred which left a strong impression on $\mathrm{my}$ mind that the action of arteries in other situations might be more simply and more effectually controlled by acupressure than by other means.

A young man had a wound in the palm of his hand, from which there was a copious hæmorrhage. Various attempts were made to secure the divided ends of the vessel. These all failed. The radial artery was tied, and the ulna artery was tied, and I believe ultimately that the brachial artery was tied; but however this may have been, the arm was at length amputated. It occurred to me that the hæmorrhage might have been restrained by means of needles passed through or underneath the bleeding vessels; and this I mentioned to a friend at the time before the amputation was performed. That such a mode of treatment might be effectual in similar cases has since been fully shown by Sir James Simpson's admirable work on "Acupressure as a Means of arresting Surgical Hæmorrhage."

I will now give a case in which $I$ had an opportunity of putting this plan of treatment into practice in a case of traumatic aneurism.

Henry G-_..., aged nineteen, admitted into St. George's Hospital on the 16th of September, 1866. On the 9th of September he received a wound on the lower part of the left popliteal space from a sharp knife. The wound at the inner side of the leg passed obliquely outwards to the extent of an inch or more. There was a great deal of hæmorrhage at the time, but this was stopped by a handkerchief being tied round the limb. The handkerchief was allowed to remain until the 12 th, when it was removed, but again applied. There was not any hæmorrhage at this time. Having removed the handkerchief on the l6th, the bleeding recurred. He was now admitted into the hospital. There was a tumour on the lower and rather to the inner side of the popliteal space about the size of a large chesnut. This could be felt and seen to distend with each arterial impulse. When the tumour was forcibly compressed by the thumb, the posterior tibial artery still pulsated. A consultation of the surgeons of the hospital was held upon the case, after which a long slender needle, previously made 
for the purpose of acupressure, was introduced immediately to the outside and above the tumour, which was at the same time pressed inward by the point of the finger. The needle was made to penetrate deeply into the popliteal space; its point was then turned inward, and brought out immediately behind the internal tuberosity of the tibia. From the grating sensation eonveyed to the fingers at this time, the needle must hare passed through some fibrous or tendinous structures. A small quantity of blood escaped at each aperture which the needle had made; this was not of a dark colour, and there was no evidence of any large vessel having been pierced. The pulsation in the tumour stopped immediately that the needle was introduced, but the pulsation in the posterior tibial artery in the lower part of the leg could still be distinctly felt. It appeared from this, and from the experiment previously made of compressing the artery, that the aneurism had arisen from a branch of the popliteal artery, and not from the popliteal itself, and that the acupressure needle must have passed between the main artery and the wounded branch. Although the tumour had ceased to beat, a piece of cork was placed immediately above it and a little to its outer side, and confined in its position by an elastic band passed over the extremities of the needle.

July 20th. - There had been a little pain up the thigh, but none near the aneurism; no pulsation in the tumour.

22nd. - The indiarubber band was removed, but the needle was allowed to remain. There was some very slight irritation at the points where the needle passed through the skin. There was no other pain or inconvenience. The skin of the leg for a considerable distance was still discoloured, presenting the appearance of having been bruised.

24th. The acupressure needle was removed (on the sixth day). Some serous fluid followed its extraction, and a very small quantity of blood. A pad of lint was now placed over the situation that the needle had occupied, and retained in its position by a bandage.

26th. - The pad and bandage were removed. Some dark, grumous-looking fluid escaped from the original wound. No pulsation in the tumour, nor fresh hæmorrhage.

27th. - The bandage was again removed, and a smaller quantity of the same kind of fluid escaped from the wound as on the preceding day.

29 th. - The wound discharged only a very small quantity of serous fluid.

Oct. 2nd.-There was now no discharge from the wound. Some thickening could be felt in the situation of the aneurism, but there was not the slightest pulsation. The pulsation in the posterior tibial artery continued natural. The skin of the leg still remained discoloured.

4th. - Feels quite well, and is in no pain. The wound has nearly healed.

6th. - Was allowed to get up.

13th. - Has had no unfavourable symptom since the last report. The discoloration of the limb has disappeared. He left the hospital apparently quite well.

The patient presented himself at the hospital on the 20th, and again on the 27 th, when, with the exception of the scars of the original wound and of the acupressure needle, the limb was perfectly in its natural condition.

This case occurred in a young man; it was a traumatic aneurism, and it was in a branch only of the main artery. It does not therefore follow that an ordinary aneurism of the popliteal artery would be cured by the same plan of treatment. On the other hand, it must be considered that this must have been a large branch, that it was very near its parent trunk, that it doubtless had its accompanying veins and nerves, and that these sustained no injury from the degree of pressure which was applied to them. Considering that an aneurism of this size and in this situation was so speedily and so completely cured by acupressure-considering that the degree of pressure required is not such as to stop the circulation, and taking into account the fact that an acupressure needle may at any moment be removed, the facts are, I think, sufficient to justify the trial of this mode of treatment in other cases of aneurism. Should increased experience confirm the a priori reasoning, there is, I think, little doubt but that this would be a simpler and more effectual way of preventing the arterial impulse than any other hitherto practised.

In conclusion, I may mention that the needles best adapted for compressing large arteries are curved, with rounded, not eutting points. These, when used, should be held firmly in a handle which can easily be remored. With a needle of this kind it is not easy to wound a large ressel in the living body. Sarile-row, October, 1866.

\section{ON SYPHILITIC AND PHAGEDENIC ULCERATION :}

THEIR PATHOLOGY AND LOCAL AND CONSTITUTIONAL TREATIIENT.

\section{Br DONALD C. BLACK, M.D., L.R.C.S. EdIr.}

(Coneluded from page 167 .)

BuT if the foregoing hypothesis be correct, seeing that the suppuration and ulceration are due to depressed vitality, and it in turn to the stagnation and consequent inadequate supply of arterial blood, the indications of correct treatment, I submit, are-1st. To allay irritability and relieve tension. Thus restore the normal function of the part, I am tempted to say, as is done by bleeding in pneumonia, or cupping and leeching in pleuritis, \&c. 2ndly. When suppuration and ulceration ensue, to imitate Nature by the application of medicaments, whose tendency it is to facilitate the due oxidation of tissue prevented in disease as above, to make way for the new arterial blood by thus indirectly stimulating absorption, raising the vitality of the part, and casting the preponderance of power in the curative hands of Nature. Fibrine, by which material repair is effected, develops itself under favourable circumstances into healthy tissue-" "1st, by solidifying into an opaquish granular mass, studded with small oval nuclei, which, like other nuclei, are rendered more distinct by acetic acid. Within a certain time the lymph acquires a distinct fibrous structure, and the nuclei probably arrange themselves into fibres."* or 2ndly, " the fibrine develops itself into a mass of large nucleated cells, which subsequently enlarge, elongate, and split into the shapes known as the caudate, fusiform, \&c.," and ultimately split up into fibrous tissue. Pus is a yellowish, opaque fluid, having a peculiar smell, and consists of a thin serum holding a vast number of globules in suspension. Under favourable circumstances, fibrine develops itself into healthy tissue without suppuration taking place; but when the vitality of the part is depressed to a certain extent, pus is formedthat is, part of the reparative material is sacrificed for the protection of the newly-forming tissue. Or to quote from Dr. Allison, "Three processes go on simultaneously in order to effect ulceration. 1st. An exudation of inflammatory lymph forms the substance of granulations. 2ndly. From a portion of the original inflammatory lymph, pus-cells are formed for the protection of the delicate granulations. 3rdly. Absorption (interstitial as well as of the débris) takes place. And the occurrence of these three phenomena constitutes ulceration. If the formation of granulations predominates, the part heals ; "but in order to its predominance, absorption and disintegration must be active. Pus then is an inferior production in the scale of organised tissue.

There is a group of diseases whose most notable manifestation or indoles is ulceration of a phagedenic nature-viz., syphilis, diphtheria, cancrum oris, erysipelas, scarlet fever (putrid), and hospital gangrene. Presenting one important feature in common, it must strike one that the remedies most successfully employed in their treatment are all members of one class, that their effects are analogous, and that indeed the one might be substituted for the other. They belong to that class of agents which, applied externally, restore healthy action, as I have already observed, by degrading, as I believe, the imperfently oxydized tissues, thus stimulating absorption, and calling into action a healthy process. Take, for example, a case of syphilitic ulceration, et ex uno disce omnes. What are the applications in use? Are they not $\mathrm{NO}_{5}$, blackwash, calomel vapour-bath, Condy's fluid, \&c.?-and they act, I submit, as already pointed out. The same observations apply to the other diseases alluded to. In diphtheria one adrocates iodide of potassinm, another tincture of the muriate of iron, a third chlorate of potash, and so on. In cancrum oris common consent will point to chlorate of potash as the most reliable therapeutic agent. Again, in scarlet fever, tincture of the muriate of iron, chlorate of potash, and hydrochloric acid are identified with the disease. In erysipelas the efficacy of the tincture of the muriate of iron is widely acknowledged ; and in hospital gangrene, nitric acid, the chlorides, Condy's fluid, and such other remedies as belong to this class, are those selected in treatment. 